\title{
Low dose cytokine and the psycho-neuro-endocrine- immune approach in dermatology: Here is the future
}

Torello Lotti ${ }^{1,2,3 *}$

${ }^{1}$ Professor and Chair of Dermatology at University of Rome “ G. Marconi”, Rome, Italy

${ }^{2}$ Director of Institute of Dermatology LifeCronos, Florence, Italy

${ }^{3}$ President of World Health Academy Foundation, Zurich, Switzerland

\section{Introduction to low dose medicine}

In recent years the research in the fields of molecular biology and physiopathology highlighted the pivotal role of signaling molecules (hormones, neuropeptides, cytokines and growth factors) in both physiological and pathological processes in clear accordance with a new unified vision of the biological functions of the body, guiding principle of Psycho-Neuro-Endocrine-Immunology (PNEI) [1-6].

The PNEI vision is centered on the role of the bi-directional crosstalk between the psychoneuroendocrine systems and the immune system managed by a complex network of signaling molecules which are the carriers of the biological information necessary for the homeostatic regulation of all cellular responses. An altered cross-talk due to an imbalance between specific signal molecules is proved to be fundamental for the onset of inflammatory, allergic and autoimmune diseases [7-9].

To preserve and/or recover the homeostatic equilibrium of PNEI axis are the expected goals of Low Dose Medicine (LDM) which suggest the use of biological molecules in order to restore the starting physiological conditions (homeostasis).

LDM represents an innovative medical approach which integrates the most recent knowledge in the fields of Molecular Biology, PNEI and ultra-low doses research.

LDM therapeutic approach is based on messenger molecules oral administration in order to:

- $\quad$ enhance a pathologically down-regulated cellular pathway administering cytokine, hormone, neuropeptides or growth factor physiologically linked with the altered signalling.

- $\quad$ re-equilibrate a biological effect according to the principle of "opposing" molecules.

Scientific literature reports that cytokines oral intake is effective in modulating immune response [10-12] but presents the critical point of a low bioavailability [13]: this pitfall is avoided using an adequate drug delivery system. The use of low doses of active molecules per os in LDM (in a range between $10^{-9}$ (nanograms) and $10^{-15}$ (femtograms)) is made possible by the application of SKA (Sequential Kinetic Activation) technology, an innovative drug delivery system which allows these ultra-low concentrations to be effective both in basic experimental assays and in clinical therapy even below the actually considered minimum effective dose.

Scientific research has validated the principles of LDM: Since 2009 a growing body of scientific publications [14-18] extended the available data regarding LDM efficacy and safety. Collected data showed the efficacy of low dose SKA cytokines treatment on Th1/Th2/Th17 switch modulation.

\section{LDM in dermatology: An innovative therapeutic approach}

The skin defense system is composed of three main levels: the skin barrier, the innate immunity, and the acquired immunity $[19,20]$. Each layer has a specific role in order to protect the body against external and internal inflammatory triggers and infectious agents. A disorder in a specific layer can reverberate on the others layer and the loss of immune skin homeostasis contributes to the pathogenesis of inflammatory skin diseases.

Numerous dermatologic diseases with an important inflammatory component are characterized by the presence of a shift in the immunological balance which is mainly reflected in an imbalance between the cytokines expressed by Th1 and Th2 lymphocyte subpopulations. The Th1/Th2 paradigm is based on the evidence that Th1 cytokines hyper-production is strictly linked with organ-specific autoimmune diseases; skin diseases such as Psoriasis, Vitiligo and Alopecia Areata fully fall back in this pathological immune plan.

Since the 70s [21] anti-cytokine therapy was studied for the treatment of autoimmune diseases mainly targeting the typical Th1 cytokines IFNs, IL- 1 and TNF- $\alpha$ and the use of opposing interleukin and antibodies was tested for Alopecia Areata, Psoriasis and Atopic Dermatis [22-24].

The use of cytokines and other signal molecules often collides with the need of systemic administration (generally $i v$ and subcutaneous injections) of high dosages of drugs which show a wide range of dosedependent side effects in addition to the pharmacological ones [25-27].

Thanks to the LDM approach and the availability of low dose SKA cytokines, growth factor hormones and neuropeptides it is now possible to use lower doses of signal molecules (in the range of pictograms and

Correspondence to: Prof. Dr. Torello Lotti MD, MD (Hon), Professor and Chair of Dermatology, University of Rome “ G. Marconi”, Rome, Italy, Tel +393286214588; Fax +390637725647; E-mail: professor@torellolotti.it

Received: January 02, 2015; Accepted: January 12, 2015; Published: January 14, 2015 
femtograms) with therapeutic results comparable to those induced by high concentrations but without connected side effects.

A multicenter double-blind placebo-controlled clinical study performed by Roberti ML, et al. highlighted the possibility of using specific low dose SKA cytokines (IL-4; IL-10; IL-11, at a concentration of $10 \mathrm{fg} / \mathrm{ml}$ GUNA S.p.a. Milan, Italy) for the therapy of Psoriasis Vulgaris.

The efficacy of treatment with low-dose cytokines was evaluated both in terms of improvement of the condition of psoriatic lesions and in the quality of life evaluated using the rating scales PASI (Psoriasis Area Severity Index) and DLQI (Dermatology Life Quality Index) for the evaluation of the extent of the lesions and to determine the quality of life respectively. The authors identified some key points on the activity of the tested cytokines on Psoriasis: low dose SKA cytokine administration is effective and safe and has a long-term action.

This feature may be crucial in view of the treatment of other chronic diseases such as vitiligo, an autoimmune dermatologic disorder characterized by skin depigmentation due to the loss of cutaneous melanocytes. In vitiligo an imbalance in cytokine expression at cutaneous lesions level is observed, probably caused by a shift of the immune system with a prevalence of Th1/Th17 (high IL-1 and IL-17 levels) and a reduction of the Tregs/Th2-driven response (low IL-4 level) TNF- $\alpha$ also has a pivotal role in oxidative stress-mediated cytotoxicity directed against melanocytes and keratinocytes $[28,29]$. Loss of melanocytes exerts a central role in Vitiligo depigmentation. Increased melanocytes death rate is due to the disruption of the crosstalk between these cells and keratinocytes.

Oxidative stress (free oxygen radicals) and other inflammatory triggers such as TNF- $\alpha$ induce keratinocytes apoptosis with consequent reduced production of keratinocytes-released factors SCF (Stem Cell Factor), ETs (Endothelins) and, above all, bFGF (basic Fibroblast Growth Factor) that negatively affects the vitality of melanocytes. Acting at the origin of the inflammatory phenomena counteracting pro-inflammatory cytokine with specific low dose SKA cytokines (IL10, Anti-IL-1 and IL-4) and, in the meantime, stimulating melanocytes to produce melanin via up-regulation of trans-membrane receptors through SKA low dose b-FGF represent the new LDM approach for Vitiligo treatment.

\section{Conclusions}

The immune imbalance between the Th1/Th2/Th17-mediated cellular responses is a pivotal point in the etiology of numerous dermatologic disorders and the management of the altered cytokine profile could represent an important therapeutic approach.

Regrettably, if the intervention with high doses of recombinant cytokines, antibodies and other signal molecules is effective against some immune aspects of treated diseases on the other hand this approach is affected by unavoidable dose-dependent and time-dependent side effects; these pitfalls are particular important considering that autoimmune and inflammatory skin diseases are often chronic.

A new opportunity for the development of therapeutic strategies based on fine tuned immune rebalance interventions is allowed by the availability of medications containing low-dose SKA-activated cytokines. Data from pre-clinical research in the field of LDM and scientific evidences of LDM approach efficacy and safety in the treatment of Psoriasis Vulgaris and the well-grounded therapeutic hypothesis based on the administration of low dose SKA cytokines for
Vitiligo allow us to speculate that LDM-based therapeutic approach represents an unique opportunity for the development of an innovative immunological treatment for dermatological diseases characterized by an immune Th1/Th2/Th17 imbalance.

\section{References}

1. Commins SP, Borish L, Steinke JW (2010) Immunologic messenger molecules: cytokines, interferons, and chemokines. J Allergy Clin Immunol 125: S53-72. [Crossref]

2. Bacchus W, Aubel D, Fussenegger M (2013) Biomedically relevant circuit-design strategies in mammalian synthetic biology. Mol Syst Biol 9: 691. [Crossref]

3. Ader R, Cohen N, Felten DL (1987) Brain, behavior, and immunity. Brain Behav Immun 1: 1-6.

4. Ader R, Felten D, Cohen N (1990) Interactions between the brain and the immune system. Annu Rev Pharmacol Toxicol 30: 561-602. [Crossref]

5. Ader R, Cohen N (1993) Psychoneuroimmunology: conditioning and stress. Annu Rev Psychol 44: 53-85. [Crossref]

6. Ader R, Cohen N, Felten D (1995) Psychoneuroimmunology: interactions between the nervous system and the immune system. Lancet 345: 99-103. [Crossref]

7. Haroon E, Raison CL, Miller AH (2012) Psychoneuroimmunology meets neuropsychopharmacology: translational implications of the impact of inflammation on behavior. Neuropsychopharmacology 37: 137-62. [Crossref]

8. Ngoc PL, Gold DR, Tzianabos AO, Weiss ST, Celedón JC (2005) Cytokines, allergy, and asthma. Curr Opin Allergy. Clin Immunol 5: 161-166. [Crossref]

9. Lourenço EV, La Cava A (2009) Cytokines in systemic lupus erythematosus. Curr Mo Med 9: 242-254. [Crossref]

10. Burnett AF, Biju PG, Lui H, Hauer-Jensen M (2013) Oral interleukin 11 as a countermeasure to lethal total-body irradiation in a murine model. Radiat Res 180 595-602. [Crossref]

11. Hanson ML, Hixon JA, Li W, Felber BK, Anver MR, et al. (2014) delivery of IL-27 recombinant bacteria attenuates immune colitis in mice. Gastroenterology 146: 210 221. [Crossref]

12. Forster K, Goethel A, Chan CW, Zanello G, Streutker C, et al. (2012) An oral CD3specific antibody suppresses T-cell-induced colitis and alters cytokine responses to T-cell activation in mice. Gastroenterology 143: 1298-307. [Crossref]

13. Renukuntla J, Vadlapudi AD, Patel A, Boddu SH, Mitra AK (2013) Approaches for enhancing oral bioavailability of peptides and proteins. Int J Pharm 447: 75-93. [Crossref]

14. Gariboldi S, Palazzo M, Zanobbio L, Dusio GF, Mauro V, et al (2009) Low dose oral administration of cytokines for treatment of allergic asthma. Pulm Pharmacol Ther 22: 497-510. [Crossref]

15. D’Amico L, Ruffini E, Ferracini R, Roato I (2012) Low Dose of IL-12 stimulates T Cel response in cultures of PBMCs derived from Non Small Cell Lung Cancer Patients. Journal of Cancer Therapy 3: 337-342. [Cross ref]

16. Cardani D, Dusio GF, Luchini P, Sciarabba M, Solimene U, et al (2013) Ora Administration of Interleukin-10 and Anti-IL-1 Antibody Ameliorates Experimental Intestinal Inflammation. Gastroenterology Research 6: 124-133. [Crossref]

17. Radice E, Miranda V, Bellone G (2014) Low-doses of sequential-kinetic-activated interferon-gamma enhance the ex vivo cytotoxicity of peripheral blood natural killer cells from patients with early-stage colorectal cancer. A preliminary study Intern. Immunopharm 19: 66-73. [Crossref]

18. Roberti ML, Ricottini L, Capponi A, Sclauzero E, Vicenti P, et al. (2014) Immunomodulating treatment with low dose Interleukin-4, Interleukin-10 and Interleukin-11 in psoriasis vulgaris. J Biol Regul Homeost Agents 28: 133-139. [Crossref]

19. Turvey SE, Broide DH (2010) Innate immunity. J Allergy Clin Immunol 125: S24-32. [Crossref]

20. Dainichi T, Hanakawa S, Kabashima K (2014) Classification of inflammatory skin diseases: A proposal based on the disorders of the three-layered defense systems, barrier, innate immunity and acquired immunity. J Dermatol Sci 76: 81-89. [Crossref]

21. Skurkovich SV, Klinova EG, Eremkina EI, Levina NV (1974) Immunosuppressive effect of an anti-interferon serum. Nature 247: 551-552. [Crossref] 
22. Trepicchio WL, Ozawa M, Walters IB. (1999) Interleukin-11 therapy selectively downregulates type I cytokine proinflammatory pathways in psoriasis lesions. $J$ Clin Invest 104: 1527-1537. [Crossref]

23. Skurkovich S, Korotky NG, Sharova NM, Skurkovich B (2005) Treatment of alopecia areata with anti-interferon-gamma antibodies. J Investig Dermatol Symp Proc 10: 283284. [Crossref]

24. Numerof RP, Asadullah K (2006) Cytokine and anti-cytokine therapies for psoriasis and atopic dermatitis. BioDrugs 20: 93-103. [Crossref]

25. Wallis RS (2007) Reactivation of latent tuberculosis by TNF blockade: the role of interferon gamma. J Investig Dermatol Symp Proc 12: 16-21. [Crossref]
26. Wallis RS, Broder M, Wong J, Lee A, Hoq L (2005) Reactivation of latent granulomatous infections by infliximab. Clin Infect Dis 41: S194-S1948. [Crossref]

27. Guerra I, Algaba A, Pérez-Calle JL, Chaparro M, Marín-Jiménez I, et al. (2012) Induction of psoriasis with anti-TNF agents in patients with inflammatory bowel disease: a report of 21 cases. Crohns Colitis 6: 518-523. [Crossref]

28. Sandoval-Cruz M, García-Carrasco M, Sánchez-Porras R, et al. (2011) Immunopathogenesis of vitiligo. Autoimmun Rev 10: 762-765. [Crossref]

29. Laddha NC(1), Dwivedi M, Mansuri MS, Gani AR, Ansarullah M, et al. (2013) Vitiligo: interplay between oxidative stress and immune system. Exp Dermatol 22: 245-250. [Crossref]

Copyright: (C2015 Lotti T. This is an open-access article distributed under the terms of the Creative Commons Attribution License, which permits unrestricted use, distribution, and reproduction in any medium, provided the original author and source are credited. 